\title{
Formulation of Highly Soluble Poly(ethylene glycol)-Peptide DNA Condensates
}

\author{
Kai Y. Kwok, Donald L. McKenzie, David L. Evers, and Kevin G. Rice* \\ Contribution from Divisions of Pharmaceutics and Medicinal Chemistry, College of Pharmacy, University of Michigan, \\ Ann Arbor, Michigan 48109-1065.
}

Received March 4, 1999. Accepted for publication July 19, 1999.

\begin{abstract}
Two poly(ethylene glycol) (PEG)-peptides were synthesized and tested for their ability to bind to plasmid DNA and form soluble DNA condensates with reduced spontaneous gene expression. PEG-vinyl sulfone or PEG-orthopyridyl disulfide were reacted with the sulfhydryl of Cys-Trp-Lys $18\left(\mathrm{CWK}_{18}\right)$ resulting in the formation of nonreducible (PEG-VS-CWK 18 ) and reducible (PEG-SS-CWK 18 ) PEGpeptides. Both PEG-peptides were prepared on a micromole scale, purified by RP-HPLC in $>80 \%$ yield, and characterized by ${ }^{1} \mathrm{H}$ NMR and MALDI-TOF. PEG-peptides bound to plasmid DNA with an apparent affinity that was equivalent to alkylated (Alk)CWK 18 , resulting in DNA condensates with a mean diameter of 80-90 nm and $\zeta$ (zeta) potential of $+10 \mathrm{mV}$. The particle size of PEG-peptide DNA condensates was constant throughout the DNA concentration range of $0.05-2 \mathrm{mg} / \mathrm{mL}$, indicating these to be approximately 20 -fold more soluble than AlkCWK ${ }_{18}$ DNA condensates. The spontaneous gene transfer to HepG2 cells mediated by PEG-VS-CWK ${ }_{18}$ DNA condensates was over two orders of magnitude lower than PEG-SS-CWK 18 DNA condensates and three orders of magnitude lower than AlkCWK ${ }_{18}$ DNA condensates. PEG-VS-CWK ${ }_{18}$ efficiently blocked in vitro gene transfer by reducing cell uptake. The results indicate that a high loading density of PEG on DNA is necessary to achieve highly soluble DNA condensates that reduce spontaneous in vitro gene transfer by blocking nonspecific uptake by HepG2 cells. These two properties are important for developing targeted gene delivery systems to be used in vivo.
\end{abstract}

\section{Introduction}

A variety of macromolecules including cationic lipids, ${ }^{1}$ polylysine, ${ }^{2}$ polyethylenimine, ${ }^{3}$ and dendrimers ${ }^{4}$ have been used as carriers to bind to negatively charged plasmid DNA and facilitate spontaneous gene transfer in cell culture as a result of charge interaction between the DNA carrier complex and the cell surface. Unfortunately, the performance of these nonviral gene delivery carriers are far less efficient in vivo due in part to the rapid pharmacokinetics and clearance of DNA complexes., 5 This relates to both the particle size and surface charge of the delivery system. ${ }^{7}$ For example, cationic lipids form large DNA complexes that are trapped in the capillary beds of the lung ${ }^{5}$ whereas smaller $(<100 \mathrm{~nm})$ peptide DNA condensates are scavenged by mononuclear phagocytic system (MPS) cells of the liver, ${ }^{6}$ limiting the development of DNA delivery systems that target peripheral tissues.

The intravenous dosing of most colloids leads to opsonization and MPS cell uptake in the lung, liver, and spleen. ${ }^{8}$ In the case of liposomes, this limitation has been largely overcome by simultaneously reducing the particle size to $<100 \mathrm{~nm}$ and modifying the surface with PEG ${ }^{9}$ since

* To whom correspondence should be addressed. Tel: 734-763-1032. Fax: 734-763-2022. E-mail: krice@umich.edu.

996 / Journal of Pharmaceutical Sciences Vol. 88, No. 10, October 1999 this polymer possesses the ideal hydration and flexibility to create a steric layer that allows liposomes to avoid opsonization and detection by MPS cells. ${ }^{10}$

Several studies have described the synthesis of PEGcontaining polymers designed to create a steric layer on the surface of DNA condensates, , 11-17 with the aim of improving their solubility and in vivo performance. An early study by Wolfert et al. described the synthesis of grafted copolymers of PEG (5 or $12 \mathrm{kDa}$ ) and polylysine ${ }_{100}$ prepared by carbodiimide coupling 5-10 mol \% of succinylated-PEG onto the side chains of polylysine. ${ }^{11,12}$ The resulting PEG-peptides were less toxic to cells in culture compared to polylysine 100 , formed DNA condensates possessing a reduced effective surface charge, and had slightly improved solubility over control polylysine DNA condensates but surprisingly were unable to reduce spontaneous gene transfer in $\mathrm{H}$ epG 2 cells ${ }^{12}$ in vitro, suggesting that they would not be able to block nonspecific interactions with cells in vivo.

Subsequently, Choi et al. derivatized the side chains of polylysine $_{120}$ with 5-25 mol \% low molecular weight PEG of $550 \mathrm{Da} .{ }^{13}$ The resulting PE G-peptide DNA condensates were also less toxic to cells than polylysine ${ }_{120}$ DNA condensates but were similar to Wolfert's PEG-peptides in their inability to reduce spontaneous gene transfer in HepG2 cells, indicating that low molecular weight PEG was no more effective than high molecular weight PEG. Using a similar approach, PEG polylysine dendrimer copolymers were recently reported by the same group and used to form DNA condensates that were more nuclease resistant than polylysine DNA condensates but which were not examined for gene transfer. ${ }^{14}$

In addition, Katayose et al. synthesized PEG-polylysine block copolymers to incorporate 5 kDa PE G into polylysine by random polymerization of $\mathrm{N}$-carboxyanhydride $\epsilon$-(benzyloxycarbonyl)-lysine with amino-PEG, resulting in a polymer with a lysine to PEG ratio of approximately 18: $1^{15,16}$ representing approximately $5 \mathrm{~mol} \%$ PEG. These PEG-polylysine DNA condensates were also reportedly much more resistant to endonuclease than uncondensed DNA but were not examined for solubility or gene transfer. ${ }^{16,17}$

To date, there are no reports of a PEG-peptide DNA condensing agents that significantly increase the solubility of the DNA condensates and/or reduce spontaneous gene transfer to HepG2 cells. These are both significant problems since the poor solubility of peptide DNA condensates of approximately $50-100 \mu \mathrm{g} / \mathrm{mL}$ limits the dosing volume and precludes dose escalation during in vivo gene transfer. Likewise, reducing the level of spontaneous gene transfer in vitro is a first step toward developing DNA formulations that avoid nonspecific gene transfer to cells in vivo and may allow the development of DNA formulations that target to peripheral sites. Here we describe the synthesis and formulation properties of two PEG-peptides, both of which 
significantly improve DNA condensate solubility and one of which dramatically reduces spontaneous gene transfer of DNA condensates in vitro. The results suggest that these two properties are closely linked to the loading level of PEG on peptide DNA condensates.

\section{Materials and Methods}

PEG-orthopyridyl disulfide (PEG-OPSS, $5 \mathrm{kDa}$ ) and PEG-vinyl sulfone (PEG-VS, $5 \mathrm{kDa}$ ) were purchased from Shearwater Inc. (Huntsville, AL) and Fluka (Ronkonkoma, NY), respectively. Fetal calf serum and LipofectAce were obtained from Gibco BRL (Gaithersburg, MD). Minimum essential media (MEM) and CM Sephadex C50 were purchased from Sigma (St. Louis, MO). TCEP (tris(2-carboxyethyl)phosphine hydrochloride) was purchased from Aldrich, (Milwaukee, WI). D-Luciferin and luciferase from Photinus pyralis were from Boehringer Mannheim (Indianapolis, IN). The $5.6 \mathrm{~kb}$ plasmid ( $\mathrm{pCMVL}$ ) encoding the reporter gene luciferase under the control of the cytomegalovirus promoter was a gift from Dr. M. A. Hickman at the University of California, Davis. ${ }^{18}$ pCMVL was produced in E. coli and purified using a Qiagen Ultrapure-100 kit (Santa Clarita, CA). Bradford reagent was purchased from Bio-Rad (Hercules, CA). Preparative and analytical C18 reverse phase HPLC columns were purchased from $V y$ dac (Hesperia, CA). HPLC was performed using a computer-interfaced HPLC and fraction collector from ISCO (Lincoln, NE).

Synthesis of PEG-VS-CWK $\mathbf{1 8}$ and PEG-SS-CWK ${ }_{\mathbf{1 8}}-\mathrm{CWK}_{18}$ (Cys-Trp-Lys 18 ) and dimeric-CWK ${ }_{18}$ were synthesized and characterized as described previously. ${ }^{19}$ The Cys residue on $\mathrm{CWK}_{18}$ was alkylated with iodoacetic acid resulting in AlkCWK 18 as reported. ${ }^{19}$ The synthesis of PEG-VS-CWK 18 utilized dimeric$\mathrm{CWK}_{18}(0.5 \mu \mathrm{mol})$ which was reduced to form $1 \mu \mathrm{mol}$ of $\mathrm{CWK}_{18}$ by reaction with $25 \mu \mathrm{mol}$ of TCEP20 in $0.5 \mathrm{~mL}$ of $0.1 \mathrm{M}$ sodium phosphate $\mathrm{pH} 7$ for $4 \mathrm{~h}$ at room temperature. PEG-VS-CWK 18 was formed by reacting $1 \mu \mathrm{mol}$ of reduced CWK 18 with $30 \mu \mathrm{mol}$ of PEGVS in a total volume of $1.2 \mathrm{~mL}$ of $0.1 \mathrm{M}$ sodium phosphate $\mathrm{pH} 7$ at room temperature for $12 \mathrm{~h}$. The progress of the reaction was monitored by analytical RP-HPLC eluted at $1 \mathrm{~mL} / \mathrm{min}$ with $0.1 \%$ TFA and a gradient of acetonitrile (5-65\% over $30 \mathrm{~min}$ ) while detecting by $A_{280 \mathrm{~nm}}$. The reaction mixture was applied to a CM Sephadex C50 cation-exchange column $(0.7 \times 15 \mathrm{~cm})$ el uted with $60 \mathrm{~mL}$ of water to remove free PEG-VS as the unbound fraction and then with $15 \mathrm{~mL}$ of $1.5 \mathrm{M}$ sodium chloride while collecting 5 $\mathrm{mL}$ fractions. PEG-VS-CWK 18 and $\mathrm{CWK}_{18}$ were detected by $\mathrm{A}_{280 \mathrm{~nm}}$ and were pooled and desalted by $5 \mathrm{~h}$ dialysis against $4 \mathrm{~L}$ of water in 1000 MWCO tubing and then freeze-dried. PEG-VS-CWK 18 was resolved from $\mathrm{CWK}_{18}$ by injecting $0.5 \mu \mathrm{mol}$ onto a semipreparative C18 RP-HPLC column $(2 \times 25 \mathrm{~cm})$ el uted at $10 \mathrm{~mL} / \mathrm{min}$ with $0.1 \%$ TFA and a gradient of acetonitrile ( 5 to $65 \%$ over $30 \mathrm{~min}$ ) while detecting by $A_{280 \mathrm{~nm}}$. The peak eluting at $25 \mathrm{~min}$ yielded $0.8 \mu \mathrm{mol}$ of PEG-VS-CWK 18 (80\%) based on tryptophan absorbance $\left(\epsilon_{280 \mathrm{~nm}}\right.$ $\left.=5600 \mathrm{M}^{-1} \mathrm{~cm}^{-1}\right)$.

A disulfide bond exchange reaction was used to prepare PEGSS-CWK 18 . Prior to conjugation of PEG-OPSS, dimeric-CWK 18 was reduced and then purified by RP-HPLC eluted as described above. Reduced CWK 18 ( $1 \mu \mathrm{mol})$ was reacted with $4 \mu \mathrm{mols}$ of PEG-OPSS in $1 \mathrm{~mL}$ of $0.1 \mathrm{M}$ sodium phosphate $\mathrm{pH} 7$ at room temperature for $30 \mathrm{~min}$. The reaction was monitored by analytical RP-HPLC which detected a single new product peak eluting at $25 \mathrm{~min}$. PEG-SS$\mathrm{CWK}_{18}$ was purified by injecting $0.5 \mu \mathrm{mol}$ portions onto semipreparative RP-HPLC eluted as described above resulting in an isolated yield of $95 \%$.

PEG-VS-CWK $_{18}$ and PEG-SS-CWK 18 ( $\left.1 \mu \mathrm{mol}\right)$ were prepared for ${ }^{1} \mathrm{H} N M R$ by $D_{2} \mathrm{O}$ exchange followed by dissolving the sample in $0.5 \mathrm{~mL}$ of $\mathrm{D}_{2} \mathrm{O}(99.96 \%)$ containing acetone as an internal standard. ${ }^{1} \mathrm{H}$ NMR spectra were generated on a Bruker $500 \mathrm{MHz}$ spectrometer operated at $23^{\circ} \mathrm{C}$. PEG-peptides were prepared for MALDI-TOF by dissolving $5 \mathrm{nmol}$ in $20 \mu \mathrm{L}$ of water. These $(0.5$ $\mu \mathrm{L}$ ) were combined with $0.5 \mu \mathrm{L}$ of saturated $\alpha$-cyano-4-hydroxycinnamic acid in $50 \mathrm{v} / \mathrm{v} \%$ acetonitrile and $0.3 \%$ trifluoroacetic acid and then analyzed on a Vestec LaserTec MS (PerSeptive Biosystems, Framingham, MA) operated in the linear mode at $20 \mathrm{kV}$.

Formulation of Peptide DNA Condensates-Peptide DNA condensates were formed by adding $75 \mu \mathrm{g}$ of DNA (pCMVL in 750 $\mu \mathrm{L}$ of $5 \mathrm{mM}$ Hepes $\mathrm{pH} 7.4$ ) to varying amounts of peptide (7.5 to $90 \mathrm{nmol}$ in $750 \mu \mathrm{L}$ of Hepes) while vortexing, followed by equilibration at room temperature for $1 \mathrm{~h}$. Peptide binding to DNA was monitored by a fluorescent dye displacement assay. ${ }^{19} \mathrm{~A} 1 \mu \mathrm{g}$ aliquot of the peptide DNA condensate was diluted to $1 \mathrm{~mL}$ in Hepes containing $0.1 \mu \mathrm{M}$ thiazole orange. The fluorescence of the intercalated dye was measured on an LS50B fluorometer (PerkinElmer, UK) in a microcuvette by exciting at $500 \mathrm{~nm}$ while monitoring emission at $530 \mathrm{~nm}$.

The particle size of peptide DNA condensates were analyzed at a DNA concentration of $50 \mu \mathrm{g} / \mathrm{mL}$ in Hepes by quasielastic light scattering (QELS). The particle surface charge was determined by $\zeta$ (zeta) potential analysis using a Brookhaven ZetaPlus (Brookhaven Instruments). The solubility of peptide DNA condensates were determined by measuring particle size as a function of DNA concentration ( $50 \mu \mathrm{g} / \mathrm{mL}$ to $2 \mathrm{mg} / \mathrm{mL}$ ) at a constant peptide: DNA stoichiometry of $0.4 \mathrm{nmol}$ of peptide per $\mu \mathrm{g}$ of DNA corresponding to a charge ratio $\left(\mathrm{NH}_{4}^{+}: \mathrm{PO}_{4}^{-}\right)$of $2.3: 1$.

DNA cocondensates were prepared by add-mixing AlkCWK 18 and PEG-VS-CWK ${ }_{18}$ in ratios ranging from 0 to $100 \mathrm{~mol} \%$ and condensing DNA at a charge ratio of 2.3:1 as described above. To establish the mol ratio of peptides bound to DNA, condensates were dialyzed in a fixed volume $(0.5 \mathrm{~mL})$ dialyzer for $75 \mathrm{~h}$ against water using a 100000 MWCO membrane. Peptide DNA condensates in the retentate $(0.5 \mathrm{~mL})$ were dissociated by adding $50 \mu \mathrm{L}$ of $5 \mathrm{M}$ sodium chloride in $0.1 \%$ TFA. AlkCWK 18 and PEG-VS$\mathrm{CWK}_{18}$ were quantified by injecting $1 \mathrm{nmol}$ of peptide $(100 \mu \mathrm{L})$ onto analytical RP-HPLC eluted with $0.1 \%$ TFA and a gradient of acetonitrile ( 5 to $65 \%$ over $30 \mathrm{~min}$ ) while detecting tryptophan by fluorescence $\left(\lambda_{\text {ex280nm }}, \lambda_{\text {em } 350 \mathrm{~nm}}\right)$. The peak integration areas were used to quantify AlkCWK 18 and PEG-VS-CWK 18 with reference to standard curves developed for each peptide.

In Vitro Gene Expression and Cell Binding-HepG2 cells were plated at $1.5 \times 10^{5}$ cells per $35 \mathrm{~mm}$ well and grown to $40-$ $70 \%$ confluence in MEM supplemented with $10 \%$ fetal calf serum (FCS). Peptide DNA condensates (10 $\mu \mathrm{g}$ of DNA) were added dropwise to triplicate sets of cells in $2 \%$ FCS containing $80 \mu \mathrm{M}$ chloroquine. After $5 \mathrm{~h}$ incubation at $37^{\circ} \mathrm{C}$, the media was replaced with MEM supplemented with $10 \%$ FCS, and luciferase expression was determined at $24 \mathrm{~h}$. Cells were washed twice with ice-cold phosphate-buffered saline (calcium and magnesium free) and then treated with $0.5 \mathrm{~mL}$ of ice-cold lysis buffer $(25 \mathrm{mM}$ Tris hydrochloride $\mathrm{pH} 7.8,1 \mathrm{mM}$ EDTA, $8 \mathrm{mM}$ magnesium chloride, $1 \%$ Triton X-100, 1 mM DTT) for $10 \mathrm{~min}$. The cell lysate was scraped, transferred to $1.5 \mathrm{~mL}$ microcentrifuge tubes, and centrifuged for $7 \mathrm{~min}$ at $13000 \mathrm{~g}$ at $4{ }^{\circ} \mathrm{C}$ to pellet debris. Lysis buffer $(300 \mu \mathrm{L})$, sodium-ATP $\left(4 \mu \mathrm{L}\right.$ of a $180 \mathrm{mM}$ solution, $\left.\mathrm{pH} 7,4^{\circ} \mathrm{C}\right)$, and cell lysate $\left(100 \mu \mathrm{L}, 4^{\circ} \mathrm{C}\right)$ were combined in a test tube, briefly mixed, and immediately placed in the luminometer. Luciferase relative light units (RLU) were recorded on a Lumat LB 9501 (Berthold Systems, Germany) with 10 s integration after automatic injection of $100 \mu \mathrm{L}$ of $0.5 \mathrm{mM}$ D-luciferin (prepared fresh in lysis buffer without DTT). The expression level of luciferase was normalized for protein using the Bradford assay, ${ }^{21}$ and the relative light units were converted to fmol of luciferase/mg of protein using a standard curve developed by adding luciferase to cell supernatant. Each experimental result represents the mean and standard deviation derived from a triplicate set of transfections.

LipofectAce (Gibco BRL, 1:2.5 w/w dimethyl dioctadecylammonium bromide and dioleoyl phosphatidylethanolamine) was optimized for use to mediate gene transfection in HepG2 cells according to the manufacturer's instructions. DNA/LipofectAce complexes were prepared by combining $10 \mu \mathrm{g}$ of DNA in $100 \mu \mathrm{L}$ of serum free media (SFM) with $60 \mu \mathrm{L}$ of LipofectAce prepared in $150 \mu \mathrm{L}$ of SFM. The LipofectAce DNA complex was then diluted with $1.7 \mathrm{~mL}$ of SFM and used to transfect HepG2 cells for $5 \mathrm{~h}$ followed by replacement of the transfecting media with MEM supplemented with $10 \%$ FBS. The cells were incubated for a total of $24 \mathrm{~h}$, harvested, and then analyzed for luciferase as described above.

Iodinated plasmid DNA was prepared with specific activity of $300 \mathrm{nCi}$ per $\mu \mathrm{g}$ of DNA as described previously.22 Prior to forming DNA condensates, the specific activity of the 125 DNA was adjusted to $4.5 \mathrm{nCi}$ per $\mu \mathrm{g}$ of DNA by combining with unlabeled plasmid. DNA condensates were prepared using AlkCWK ${ }_{18}$, PEG$\mathrm{SS}^{-C W K_{18}}$, or PEG-VS-CWK 18 as described above. Peptide 125IDNA condensates $(10 \mu \mathrm{g})$ were used to transfect HepG2 cells for $5 \mathrm{~h}$ according to the procedure described above. The radioactive media was removed, cells were washed with phosphate-buffered saline, harvested with lysis buffer, and the cell-associated radioactivity was quantified by gamma counting. 

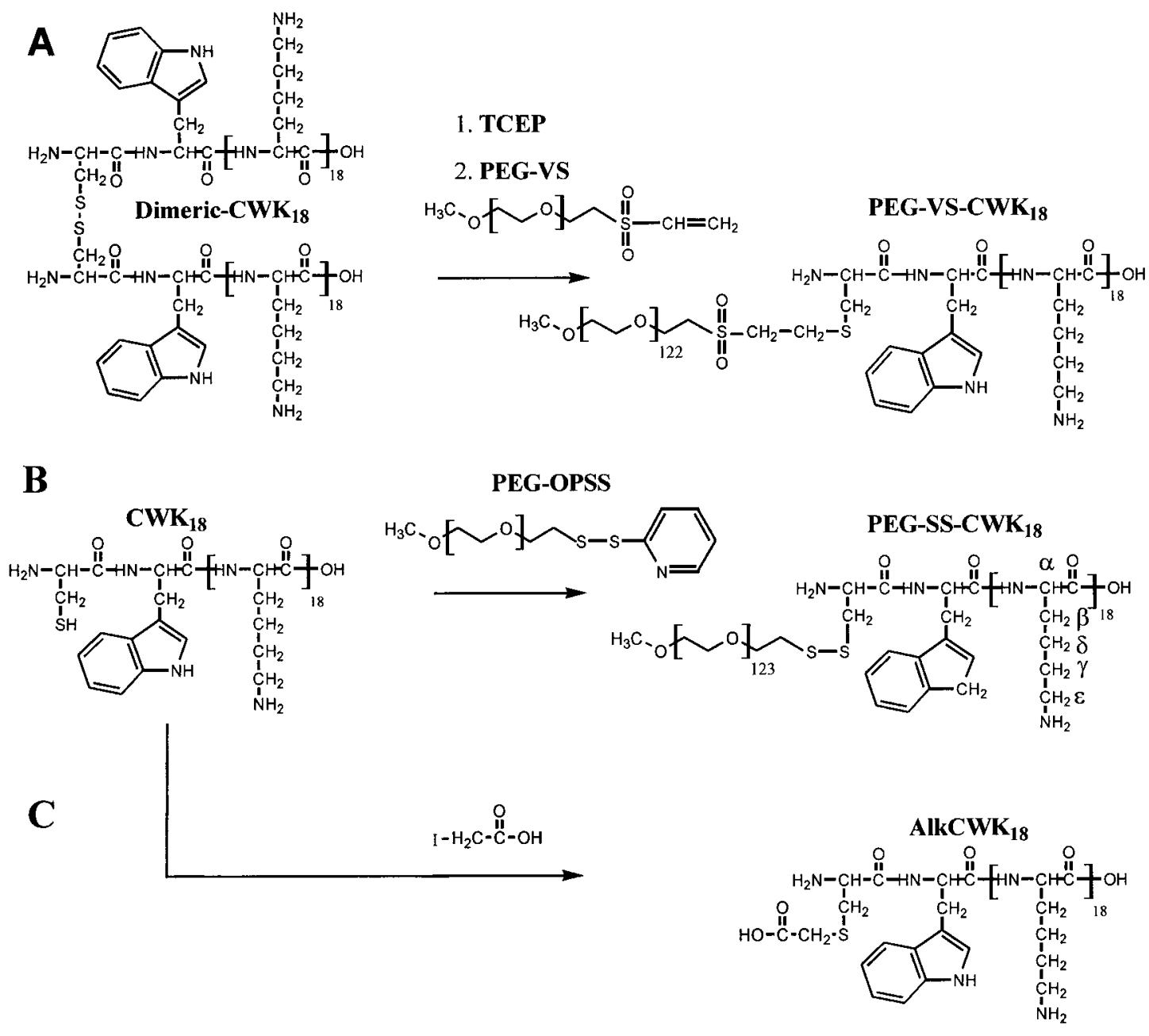

Figure 1-Reaction schemes for the synthesis of PEG-CWK ${ }_{18}$ conjugates. (A) TCEP was used to reduce dimeric-CWK 18 to generate $\mathrm{CWK}_{18}$. This was reacted in situ with PEG-VS to form PEG-VS-CWK ${ }_{18}$. (B) Alternatively, the reaction of CWK ${ }_{18}$ with PEG-OPSS formed PEG-SS-CWK 18 . (C) AlkCWK 18 was produced by reacting $\mathrm{CWK}_{18}$ with iodoacetic acid. The $\alpha, \beta, \gamma, \delta$, and $\epsilon$ protons of Lys of PEG-SS-CWK 18 illustrate the nomenclature used for assigning PEG-peptides in Figure 3 .

\section{Results}

PEG-Peptide Synthesis-PEG was covalently attached to the Cys residue of a 20 amino acid synthetic peptide $\left(\mathrm{CWK}_{18}\right)$ to prepare two PEG-peptides possessing either a reversible (PEG-SS-CWK ${ }_{18}$ ) or irreversible (PEG-VS$\mathrm{CWK}_{18}$ ) covalent linkage (Figure 1 ). Each reaction was optimized by systematically changing the $\mathrm{pH}$ and the stoichiometry of peptide to PEG while monitoring the product formation by analytical RP-HPLC. Since the reaction of $\mathrm{CWK}_{18}$ with PE G-VS at pH 7 was slow (12 h), TCEP was added to reduce dimeric- $\mathrm{CWK}_{18}$ and also inhibit its re-formation during conjugation with PEG-VS. At pH 7, a mol ratio of $P E G-V S: C W K_{18}$ of $30: 1$ resulted in optimal conjugation to form PEG-VS-CWK ${ }_{18}$. At suboptimal stoichiometries or lower $\mathrm{pH}$ the reaction was incomplete whereas at a higher $\mathrm{pH}$, dimeric- $\mathrm{CWK}_{18}$ re-formed as the major product.

In contrast to the synthesis of PEG-VS-CWK ${ }_{18}$, the optimal reaction conditions to prepare PEG-SS-CWK 18 only required a 4 mol excess of PEG-OPSS over $\mathrm{CWK}_{18}$ at $\mathrm{pH}$ 7. In this case, attempts to block the formation of dimeric$\mathrm{CWK}_{18}$ with TCEP led to the reduction of PEG-OPSS, completely inhibiting the desired reaction. I nstead, reduced $\mathrm{CWK}_{18}$ was prepared and found to react rapidly (30 min) with PEG-OPSS with minimal formation of dimeric-CWK ${ }_{18}$.

RP-HPLC analysis of the crude reaction product of PEGVS-CWK ${ }_{18}$ demonstrated a nearly complete disappearance of $\mathrm{CWK}_{18}$ with the formation of a new peak eluting at 25 min (Figure 2B). Despite the apparent complete resolution of the desired product, careful examination revealed that PEG-VS coeluted with PEG-VS-CWK ${ }_{18}$. This was evident from NMR analysis which determined a 10-fold excess of PEG relative to $\mathrm{CWK}_{18}$ in the HPLC purified product (data not shown). Consequently, PEG-VS-CWK ${ }_{18}$ was purified using cation exchange to remove excess PEG-VS and then by RP-HPLC to remove unreacted $\mathrm{CWK}_{18}$ resulting in a product that rechromatographed as a single peak on RPHPLC (Figure 2D). Proton NMR analysis identified resonances assigned to the $\alpha, \beta, \gamma, \delta$, and $\epsilon$ protons of the Lys residues as well as the Trp aromatic resonances (Figure 3A). I ntegration of protons at $3.67 \mathrm{ppm}$ (PEG) relative to the signal at $2.97 \mathrm{ppm}$ (Lys $\epsilon$ ) produced a peak area ratio of $13.5: 1$ corresponding to a 1:1 conjugate of $P E_{122}$ and $\mathrm{CWK}_{18}$.

RP-HPLC analysis of the crude reaction product of PE GOPSS and CWK 18 identified a product peak eluting at 25 min, a PE G-OPSS reagent peak at $28 \mathrm{~min}$, a thiol pyridine (TP) byproduct peak at $5 \mathrm{~min}$, and a trace of dimeric-CWK ${ }_{18}$ eluting at $15 \mathrm{~min}$ (Figure 2C). PEG-SS-CWK ${ }_{18}$ was isolated in a single step by semipreparative RP-HPLC, rechromatographed as a single peak on analytical HPLC (Figure 2E), and produced an NMR spectrum with an integration ratio of PEG:Lys also establishing a 1:1 conjugate of $P E G_{123}$ and $\mathrm{CWK}_{18}$ (Figure 3B).

MALDI-TOF analysis of PEG-VS-CWK 18 and PEG-SS$\mathrm{CWK}_{18}$ produced a broad peak centered at 8433 and 8297 


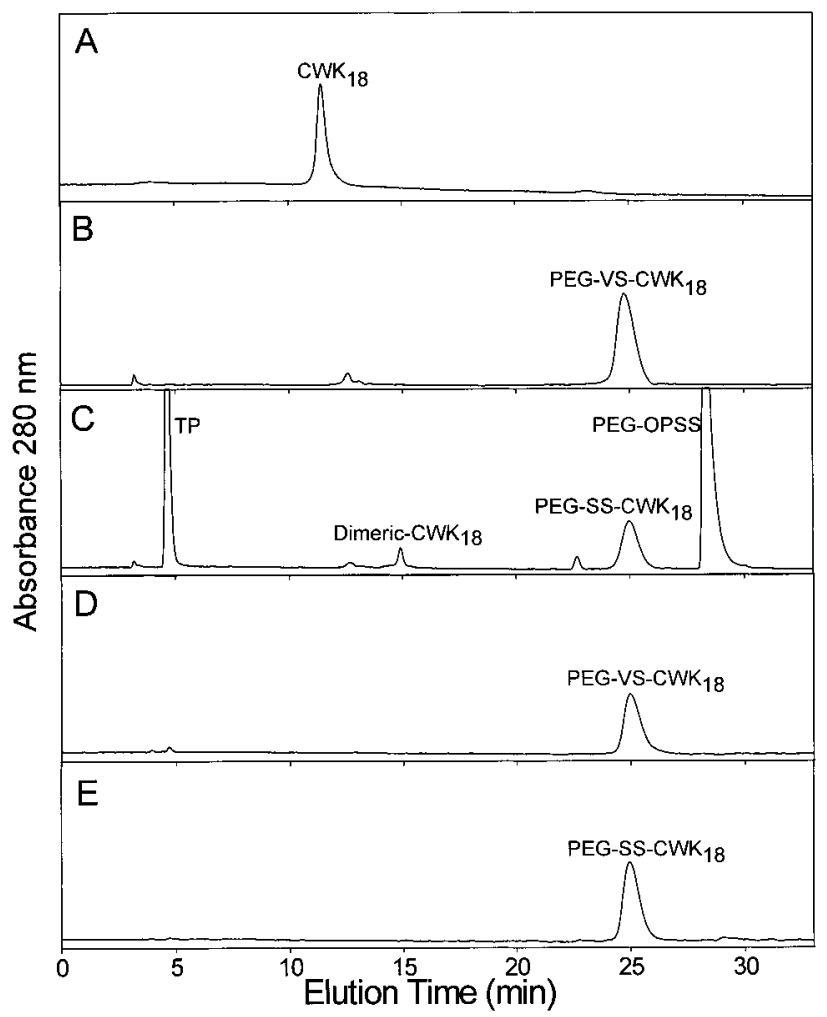

Figure 2-Analytical RP-HPLC analysis of PEG-CWK ${ }_{18}$ conjugates. The reaction of dimeric-CWK ${ }_{18}$ with TCEP and PEG-VS formed a single major product as shown in panel $B$. Alternatively, reduced $\mathrm{CWK}_{18}$ (panel A) reacted with PEG-OPSS to produce PEG-SS-CWK ${ }_{18}$, dimeric-CWK ${ }_{18}$, thiol-pyridine (TP), and unreacted PEG-OPSS as shown in panel C. Purified PEG-VS$\mathrm{CWK}_{18}$ and PEG-SS-CWK 18 both eluted as a single peak as shown in panels $D$ and $E$, respectively.
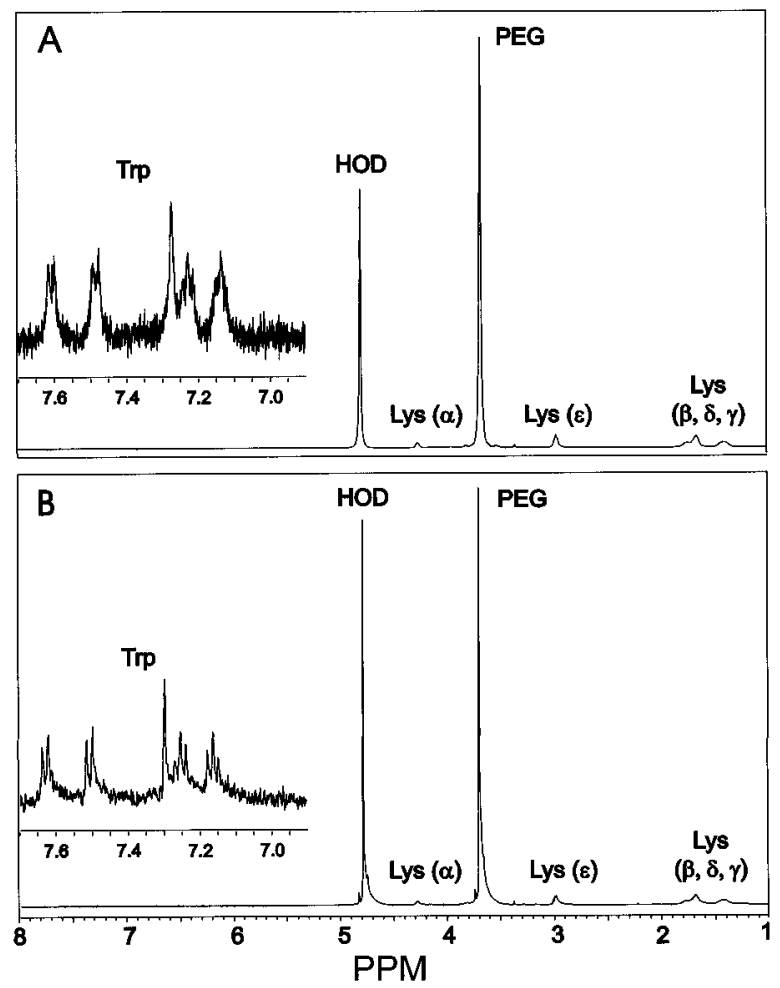

Figure $3{ }^{1}{ }^{1} \mathrm{H}$ NMR analysis of PEG-CWK ${ }_{18}$ conjugates. The $500 \mathrm{MHz}{ }^{1} \mathrm{H}$ NMR spectrum of PEG-VS-CWK 18 (panel A) and PEG-SS-CWK ${ }_{18}$ (panel B) are illustrated with the key signals of the Lys, Trp and PEG identified according to Figure 1. The integration of the $\epsilon$ protons of Lys relative to the PEG protons established a degree of polymerization of 122 for PEG-VS-CWK ${ }_{18}$ and 123 for PEG-SS-CWK 18 .

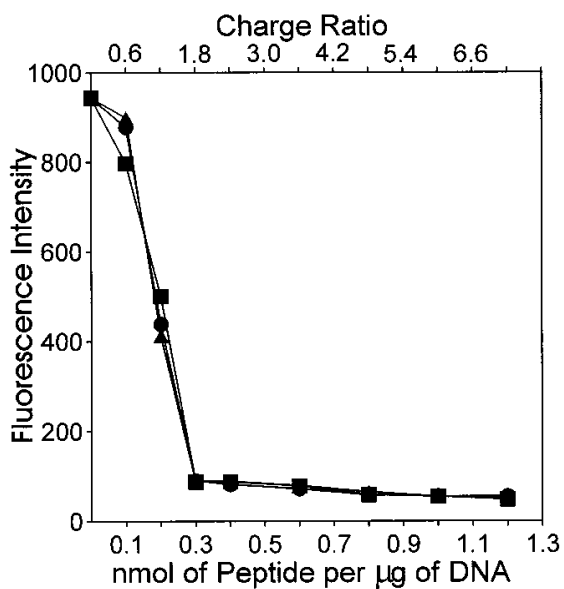

Figure 4-Relative binding affinity of PEG-CWK ${ }_{18}$ conjugates to DNA. The fluorescence intensity resulting from the titration of $\mathrm{AlkCWK}_{18}(\mathbf{O})$, PEG-SS$\mathrm{CWK}_{18}(\boldsymbol{\square})$, and PEG-VS-CWK ${ }_{18}(\boldsymbol{\Delta})$ to compete for intercalator dye binding to DNA is shown. An asymptote at $0.3 \mathrm{nmol}$ of each peptide per $\mu \mathrm{g}$ of DNA established that each peptide binds to DNA with equivalent affinity.

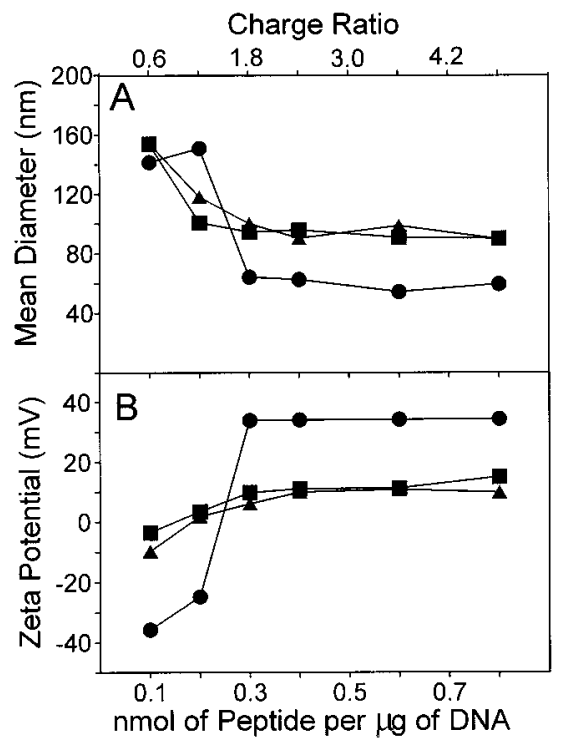

Figure 5-QELS particle size and $\zeta$ potential analysis of PEG-CWK ${ }_{18}$ DNA condensates. The mean particle size of $\operatorname{AlkCWK}_{18}(\mathbf{\bullet}), \mathrm{PEG}-\mathrm{SS}-\mathrm{CWK}_{18}(\boldsymbol{\square})$, and PEG-VS-CWK 18 ( $\mathbf{\Delta})$ DNA condensates is plotted as a function of peptide: DNA stoichiometry in panel A. The mean $\xi$ potential for each DNA condensate is plotted in panel $\mathrm{B}$. An indistinguishable particle size and $\xi$ potential was determined for each PEG-CWK 18 conjugate. However, a significant decrease in the $\xi$ potential for PEG-CWK ${ }_{18}$ DNA condensates $(+10 \mathrm{mV})$ versus AlkCWK ${ }_{18}$ DNA condensates $(+35 \mathrm{mV})$ provided evidence of the formation of a steric barrier.

$\mathrm{m} / \mathrm{z}$, respectively. These results were consistent with the formation of conjugates of $\mathrm{CWK}_{18}$ (2648 amu) and polydisperse PEG of approximately $5800 \mathrm{Da}$.

PEG-Peptide DNA Condensate Formulation-The DNA binding affinity of AlkCWK ${ }_{18}$, PEG-VS-CWK ${ }_{18}$, and PEG-SS-CWK 18 were compared using a fluorescent dye displacement assay. A coincident titration curve for each peptide with an asymptote at $0.3 \mathrm{nmol}$ per $\mu \mathrm{g}$ of DNA corresponding to a charge ratio of 1.8:1 suggested that both PEG-peptides bind to DNA with equivalent affinity as AlkCWK $_{18}$ (Figure 4).

The particle size and $\xi$ potential of DNA condensates prepared with AlkCWK ${ }_{18}$, PEG-VS-CWK 18 , and PEG-SS$\mathrm{CWK}_{18}$ were examined as a function of peptide:DNA stoichiometry (F igure 5). The mean diameter for both PEGpeptide DNA condensates was $90 \mathrm{~nm}$ at a charge ratio of 1.8:1 or higher whereas the mean diameter for AlkCWK 18 


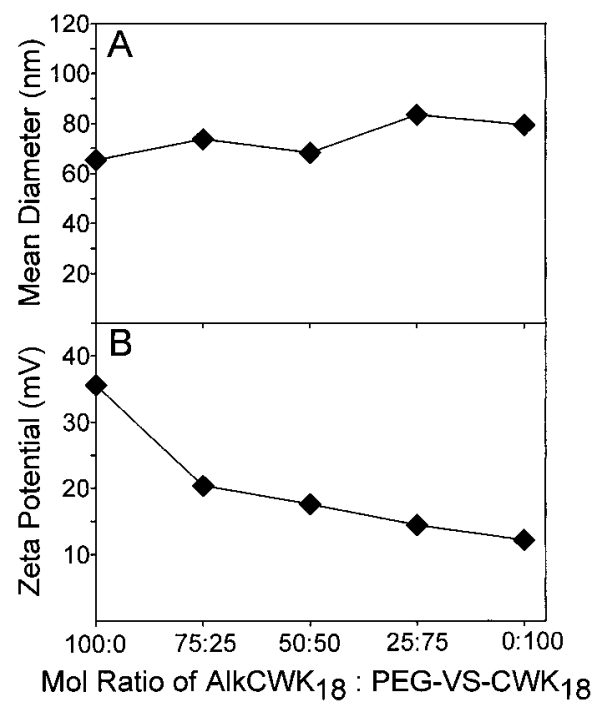

Figure $6-$ QELS particle size and $\zeta$ potential analysis of peptide DNA cocondensates. Particle size analysis was used to characterize peptide DNA cocondensates prepared at $50 \mu \mathrm{g} / \mathrm{mL}$ of DNA and varying $\mathrm{mol} \%$ of AlkCWK and PEG-VS-CWK ${ }_{18}$ as shown in panel $A$. The $\zeta$ potential of DNA cocondensates is shown in panel $B$. The mean particle size changes from 65 to $80 \mathrm{~nm}$ whereas the $\zeta$ potential of DNA cocondensates decreases from +35 to $+10 \mathrm{mV}$ with increasing $\mathrm{mol} \%$ of PEG-VS-CWK ${ }_{18}$.
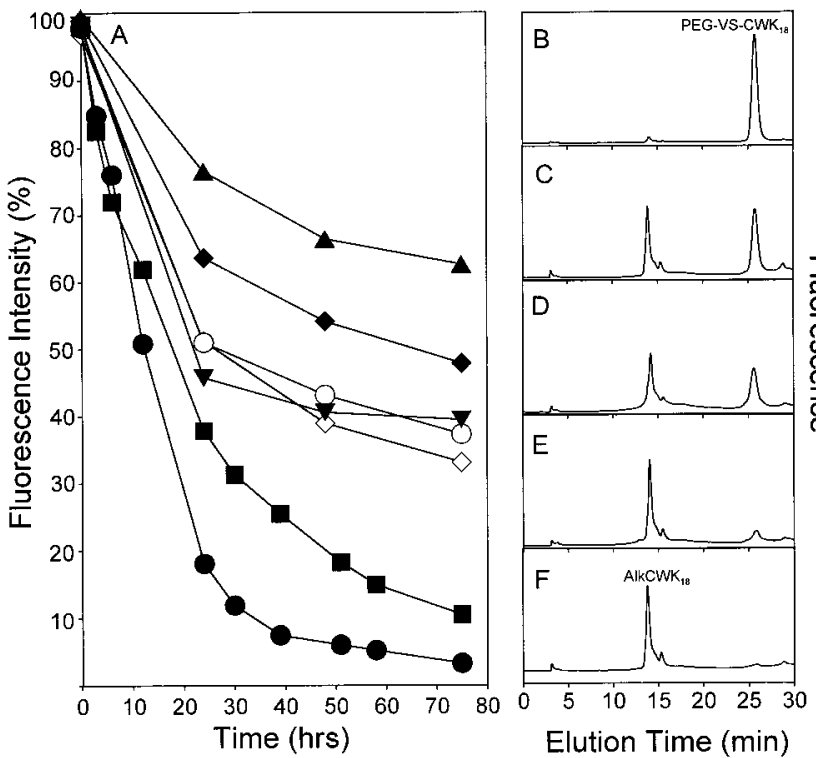

Figure 7-RP-HPLC analysis of peptide DNA cocondensates. The time course of dialysis of free AlkCWK $18(\mathbf{0})$, free PEG-VS-CWK ${ }_{18}(\boldsymbol{\square})$, AlkCWK 18 DNA condensates $(\boldsymbol{\nabla})$, PEG-VS-CWK ${ }_{18}$ DNA $(\boldsymbol{\Delta})$, and cocondensates of 25:75 ( $\bullet$ ), 50:50 (O), $75: 25(\diamond)$ mol \% of AlkCWK 18 :PEG-VS-CWK 18 bound to DNA was determined by tryptophan fluorescence of the retentate (panel A). After $75 \mathrm{~h}$ of dialysis, peptide DNA condensates in the retentate were dissociated with sodium chloride and directly chromatographed on RP-HPLC. Panels B-F illustrate chromatograms resulting from $100 \mathrm{~mol} \%$ PEG-VS-CWK ${ }_{18}$ DNA condensates (panel B), DNA cocondensates prepared with 75:25 (panel C), 50:50 (panel D), 25:75 (panel E) PEG-VS-CWK 18 :AlkCWK 18 , and $100 \mathrm{~mol} \%$ AlkCWK $_{18}$ DNA condensates (panel F).

DNA condensates was $60 \mathrm{~nm}$ (Figure 5A). In contrast, a large decrease in $\zeta$ potential of $+25 \mathrm{mV}$ was identified for PEG-peptide DNA condensates at a charge ratio of 1.8:1 compared to AlkCWK ${ }_{18}$ DNA condensates (Figure 5B).

Since PE G-VS-CWK 18 and AlkCWK 18 possess equivalent DNA binding affinity, add-mixtures of the two peptides were used to prepare DNA cocondensates. The average particle size increased from 65 to $80 \mathrm{~nm}$ using add-mixtures of AlkCWK 18 and PEG-VS-CWK 18 varying from 0 to 100 mol \% while keeping the charge ratio constant at 2.3:1
Table 1-Quantitative Analysis of DNA Cocondensates

\begin{tabular}{|c|c|}
\hline $\begin{array}{c}\text { input add-mixture } \\
\text { (mol \% AlkCWK } \text { m }_{18}: \\
\text { mol \% PEG-VS-CWK }\end{array}$ & $\begin{array}{c}\text { recovery ratio }^{a} \\
\text { (mol \% AlkCWK }{ }_{18}: \\
\text { mol \% PEG-VS-CWK }\end{array}$ \\
\hline $0: 100$ & $0: 100$ \\
\hline $25: 75$ & $41: 59$ \\
\hline $50: 50$ & $59: 41$ \\
\hline $75: 25$ & $82: 18$ \\
\hline $100: 0$ & $100: 0$ \\
\hline
\end{tabular}

${ }^{a}$ Based on HPLC standard curves developed for AlkCWK ${ }_{18}$ and PEG-VS$\mathrm{CWK}_{18}$.

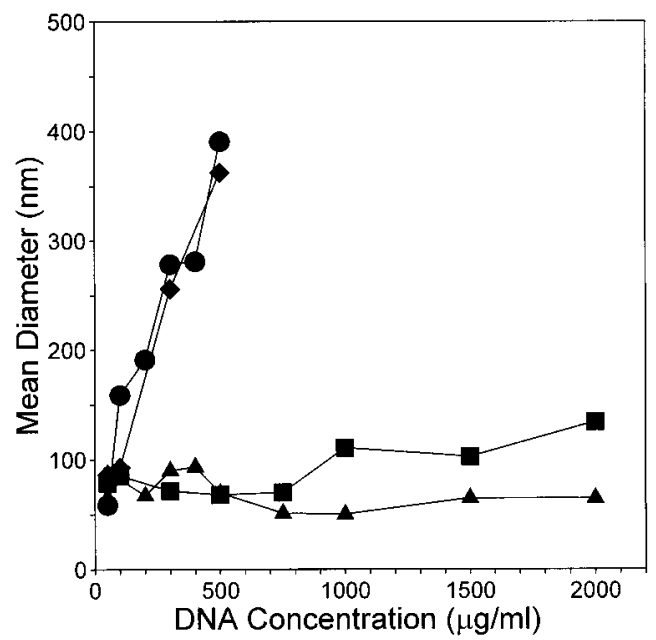

Figure 8-Solubility of peptide DNA condensates. Particle size analysis was performed as a function of DNA concentration using $100 \mathrm{~mol} \%$ AlkCWK $_{18}$ (๑) and $100 \mathrm{~mol} \%$ PEG-VS-CWK ${ }_{18}$ (ム) DNA condensates and using AlkCWK $18: P E G-V S-C W K_{18}$ DNA cocondensates prepared with $50(\diamond)$ and 90 (口) $\mathrm{mol} \%$ PEG-VS-CWK ${ }_{18}$. The particle size increased to $>400 \mathrm{~nm}$ above $500 \mu \mathrm{g} / \mathrm{mL}$ for AlkCWK ${ }_{18}$ DNA condensates but remained at $<100 \mathrm{~nm}$ for PEG-VS-CWK ${ }_{18}$ DNA condensates throughout concentrations up to $2 \mathrm{mg} /$ $\mathrm{mL}$.

(Figure 6A). Likewise, the $\zeta$ potential decreased from +35 $\mathrm{mV}$ to $+10 \mathrm{mV}$ as the stoichiometry of PEG-VS-CWK 18 increased (Figure 6B), suggesting the formation of DNA cocondensates with intermediate PEG loading.

To further confirm the formation of DNA cocondensates, unbound peptides were removed by microdialysis and the ratio of peptides bound to DNA was determined by HPLC. Control experiments established the nearly complete removal (>90\%) of free AlkCWK 18 or PEG-VS-CWK 18 from the retentate after $75 \mathrm{~h}$ of dialysis (Figure 7A). However, the dialysis of DNA cocondensates prepared at charge ratios of 2.3:1 resulted in the removal of unbound peptide and retention of $>35 \%$ of the tryptophan fluorescence. Dissociation and RP-HPLC analysis of the retained peptide (Figure 7B-F) allowed recovery of AlkCWK 18 and PEGVS-CWK 18 at ratios that agreed to within $16 \%$ of the input ratio for each DNA cocondensate (Table 1 ) in which the Ioss of PEG-VS-CWK ${ }_{18}$ was greater than that of AlkCWK ${ }_{18}$.

DNA condensate solubility was evaluated by examining the particle size of concentrated solutions. AlkCWK ${ }_{18}$ DNA condensates increased in particle size from 60 to $400 \mathrm{~nm}$ when increasing DNA concentration from 50 to $500 \mu \mathrm{g} / \mathrm{mL}$ and then formed visible flocculates at higher concentrations. Alternatively, PEG-VS-CWK ${ }_{18}$ DNA condensates maintained a mean diameter of $<100 \mathrm{~nm}$ throughout concentrations ranging from 0.05 to $2 \mathrm{mg} / \mathrm{mL}$ and showed no sign of increasing in size (Figure 8). Likewise, substitution of PEG-SS-CWK ${ }_{18}$ for PE G-VS-CWK 18 resulted in the formation of DNA condensates with $88 \mathrm{~nm}$ mean diameter at $2 \mathrm{mg} / \mathrm{mL}$. 


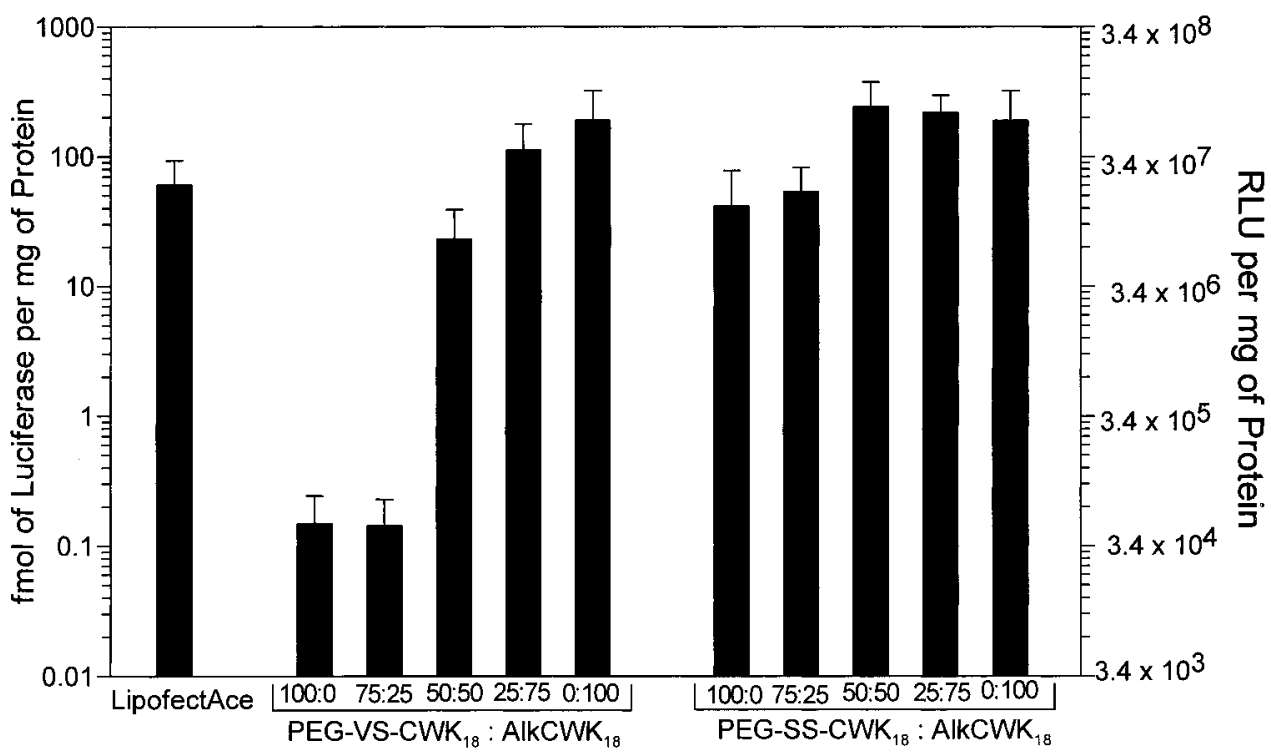

Figure 9-In vitro gene transfer efficiency of PEG-CWK ${ }_{18}$ DNA condensates. The in vitro expression of luciferase in HepG2 cells is compared for PEG-VS-CWK ${ }_{18}$ and PEG-SS-CWK ${ }_{18}$ as well as cocondensates prepared with AlkCWK ${ }_{18}$ at the ratios indicated. LipofectAce is included as a control gene transfer agent.

DNA cocondensates containing 50 mol \% PEG-VS-CWK 18 and AlkCWK 18 possessed similar poor solubility properties to that of $100 \mathrm{~mol} \% \mathrm{AlkCWK}_{18}$ DNA condensates. However, DNA cocondensates composed of 90 mol \% PEG-VS$\mathrm{CWK}_{18}$ and $10 \mathrm{~mol} \% \mathrm{AlkCWK}_{18}$ also maintained a particle size of $<100 \mathrm{~nm}$ up to $750 \mu \mathrm{g} / \mathrm{mL}$ and then formed larger particles (> $100 \mathrm{~nm}$ ) at DNA concentrations of $1 \mathrm{mg} / \mathrm{mL}$ or higher (Figure 8).

DNA condensates prepared with PEG-VS-CWK ${ }_{18}$, PEGSS-CWK ${ }_{18}$, AlkCWK $_{18}$, and add-mixtures of AlkCWK ${ }_{18}$ and PEG-peptides were compared by measuring luciferase expression in HepG2 cells $24 \mathrm{~h}$ post-transfection (Figure 9). PE G-VS-CWK ${ }_{18}$ DNA condensates reduced spontaneous gene transfer by three orders of magnitude compared to AlkCWK $_{18}$ DNA condensates. The reduction was only 10fold when transfecting with DNA cocondensates prepared with $50 \mathrm{~mol} \%$ PEG-VS-CWK 18 and only 2-fold using cocondensates composed of $25 \mathrm{~mol} \%$ PEG-VS-CWK 18 (Figure 9).

The gene transfer properties of PEG-SS-CWK 18 DNA condensates were significantly different than PEG-VS$\mathrm{CWK}_{18}$ DNA condensates. DNA condensates prepared with 100 or 75 mol \% PEG-SS-CWK 18 reduced spontaneous gene transfer by 5 -fold relative to $\mathrm{AlkCWK}_{18}$ DNA condensates while DNA cocondensates prepared with 50 or $25 \mathrm{~mol} \%$ of PEG-SS-CWK 18 were equivalent to $\mathrm{AlkCWK}_{18}$ DNA condensates (Figure 9).

The cell binding of 125 -DNA was compared for AlkCWK PEG-VS-CWK 18 , and PEG-SS-CWK 18 DNA condensates during a $5 \mathrm{~h}$ transfection. Approximately $14 \%$ of the radioactivity was cell-associated for $\mathrm{AlkCWK}_{18}$ DNA condensates whereas only $6.8 \%$ and $0.2 \%$ were cell-associated when using PEG-SS-CWK 18 and PEG-VS-CWK 18 as DNA condensing agents, respectively (F igure 10). These results correlated well with the observed gene transfer efficiency for each peptide DNA condensate, suggesting differences in the uptake of these condensates was the main cause of their difference in gene expression.

\section{Discussion}

The targeting of DNA to specific cells in vivo for the purpose of mediating therapeutically relevant levels of gene expression will require systematic optimization of the drug delivery system. ${ }^{23}$ The design of such delivery systems must

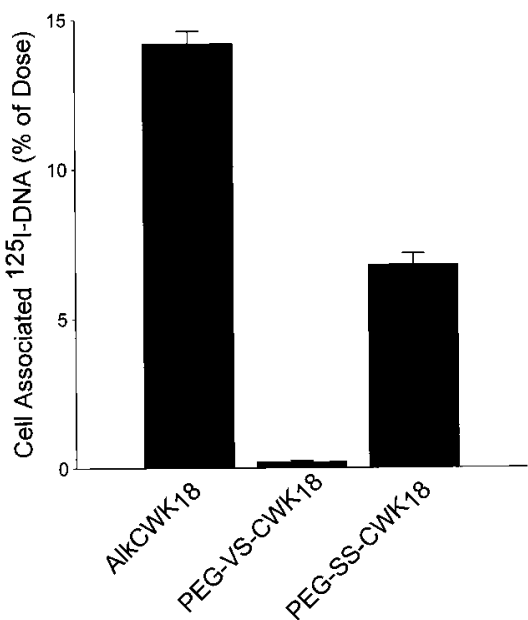

Figure 10-HepG2 cell binding of peptide DNA condensates. HepG2 cells were transfected for $5 \mathrm{~h}$ with $45 \mathrm{nCi}(10 \mu \mathrm{g})$ of ${ }^{125}$-DNA condensates prepared with either AlkCWK ${ }_{18}$, PEG-VS-CWK 18 , or PEG-SS-CWK ${ }_{18}$. The cell associated DNA recovered is expressed as the percent of ${ }^{125}$-DNA dosed onto cells. The results represent the mean \pm SD for three determinations.

attempt to minimize the carrier's toxicity and antigenicity, increase the DNA's metabolic stability, control the particle size and charge, and increase the DNA condensate solubility as well as provide a means to target DNA to the nudeus of the cell. Thus far, PEG-peptides have been reported to decrease toxicity, ${ }^{11-13}$ increase DNA stability, 14,17 and improve DNA solubility. ${ }^{12}$ In the present study we report the synthesis of two PEG-peptides that simultaneously create very soluble DNA condensates and significantly inhibit spontaneous gene transfer of peptide DNA condensates in vitro. A major finding is that both of these properties are influenced to different degrees by the load level of PEG on DNA condensates.

The directed synthesis of the low molecular weight PEGpeptides reported here is a major distinction of this work compared to others. The conjugation of PEG (5000 Da) to a single cysteine of $\mathrm{CWK}_{18}$ afforded highly purified PEGpeptides that controlled the PEG attachment site and allowed comparison of reducible and nonreducible linkages. Notably, the apparent binding affinity of both PEGpeptides for DNA were equivalent to AlkCWK $_{18}$ as determined by the intercalator exclusion assay (Figure 4), 
indicating that conjugates of $\mathrm{CWK}_{18}$ retain their ability to bind and condense DNA. Due to the homogeneity of the peptide portion of PEG-VS-CWK 18 and PEG-SS-CWK ${ }_{18}$, DNA condensates formed at charge ratios of 1.8:1 or higher achieved a constant particle size and $\xi$ potential, establishing both the absence of interfering peptides and that excess PEG-peptide does not bind to fully condensed DNA.

$\zeta$ potential measurements revealed evidence that PEGpeptides altered the surface properties of DNA condensate. The $\zeta$ potential of PEG-VS-CWK 18 and PEG-SS-CWK 18 DNA condensates were indistinguishable and reached a minimum of $+10 \mathrm{mV}$ at a calculated charge ratio of 1.8:1 (Figure 5B). The decrease in $\zeta$ potential resulted from the covalent attachment of PEG since the addition of equivalent amounts of free PEG to AlkCWK ${ }_{18}$ DNA condensates did not influenceits $\xi$ potential (data not shown). Likewise, analysis of DNA cocondensates established a correlation between the $\zeta$ potential and the mol \% of PEG-VS-CWK ${ }_{18}$ incorporated into the cocondensate (Figure 6B). The re covery of the approximate input ratio of AlkCWK 18 and PEG-VS-CWK 18 following prol onged dialysis confirmed the formation of DNA cocondensates. This allowed add-mixing of two condensing peptides (AlkCWK 18 and PEG-VS$\mathrm{CWK}_{18}$ ) to systematically alter both physical and biological properties of peptide DNA condensates.

The formation of a steric layer is highly dependent on the amount PEG loaded onto DNA condensates. ${ }^{10,12}$ This is most evident with DNA cocondensates possessing be tween 0 and 25 mol \% PEG-peptide where the $\xi$ potential decreased sharply to $+20 \mathrm{mV}$ and then only declined gradually to reach $+10 \mathrm{mV}$ at $25-100 \mathrm{~mol} \%$ PEG-peptide. Even though the $\xi$ potential only changed by $+10 \mathrm{mV}$ when titrating between 25 and $100 \mathrm{~mol} \%$ of PE G-peptide (Figure $6 \mathrm{~B})$, these DNA condensates were most al tered in solubility and gene transfer efficiency.

The solubility achieved for 100 mol \% PEG-peptide DNA condensates $(2 \mathrm{mg} / \mathrm{mL})$ is far greater than the solubility reported $(60 \mu \mathrm{g} / \mathrm{mL})$ for other PEG-peptides DNA condensates. ${ }^{12}$ This physical property appears to be very dependent on the loading density of PEG since cocondensates prepared with 50 mol \% PEG-VS-CWK ${ }_{18}$ were not improved in solubility relative to AlkCWK ${ }_{18}$ DNA condensates. Even cocondensates formed with as much as 90 mol \% PEG-VS$\mathrm{CWK}_{18}$ demonstrated an increase in particle size at concentrations greater than $750 \mu \mathrm{g} / \mathrm{mL}$, indicating that even slightly less PEG on the DNA condensate will result in lower solubility.

Earlier studies demonstrated that the in vitro gene transfer efficiency for peptide DNA condensates was dependent on the charge ratio. 19,24 The expression reached a maximum when AlkCWK ${ }_{18}$ DNA condensates were formed at charge ratio of 1.8:1 or higher, suggesting that the positive charge on DNA condensates contributes to their spontaneous transfection in cell culture. In support of this hypothesis, fully condensed PEG-VS-CWK ${ }_{18}$ DNA condensates prepared at a charge ratio of 2.3:1 possess a lower $\zeta$ potential of $+10 \mathrm{mV}$ and reduced spontaneous gene transfer by 1000 -fold compared to AlkCWK ${ }_{18}$ DNA condensates. Likewise, DNA cocondensates possessing intermediate $\xi$ potential partially reduced gene transfer, further demonstrating a correlation between DNA condensate charge and the level of spontaneous gene transfer. However, these data also established that the PEG load level needed to block spontaneous gene transfer in vitro is much lower than that required to create soluble PE G-peptide DNA condensates.

Even though PEG-VS-CWK 18 and PEG-SS-CWK ${ }_{18}$ DNA condensates were equivalent in their physical properties, they proved to be unequivalent in their ability to reduce spontaneous gene transfer (Figure 9). Since the two peptides only differ in their linkage between PEG and peptide, a possible explanation was the reduction of PEG-SS-CWK 18 either outside or inside the cell to form $\mathrm{CWK}_{18}$ DNA condensates during the time of transfection. To test this hypothesis, radi oi odinated DNA condensates were used to determine the percent cell associated after a $5 \mathrm{~h}$ transfection (Figure 10). PEG-VS-CWK ${ }_{18}$ DNA condensates did not significantly bind to cells, with only $0.2 \%$ of the dose being cell associated. In contrast, $14 \%$ of AlkCWK 18 DNA condensates and $6.8 \%$ of PEG-SS-CWK ${ }_{18}$ DNA condensates dose were cell-associated following $5 \mathrm{~h}$ transfection, supporting the hypothesis that differences in cell uptake are responsible for the 2 orders of magnitude difference in gene expression mediated by PEG-SS-CWK 18 and PEG-VS$\mathrm{CWK}_{18}$ DNA condensates. To determine if PEG-SS-CWK 18 underwent reduction during gene transfer, its stability was analyzed while incubating in cell culture media containing $2 \%$ FCS. This led to its partial reduction over time (data not shown), suggesting that the removal of PEG by disulfide bond scission results in the formation of $\mathrm{CWK}_{18}$ DNA condensates in situ and is a likely explanation of the difference between PEG-SS-CWK 18 and PEG-VS-CWK 18 DNA condensates.

Therefore, the utility of PEG-SS-CWK ${ }_{18}$ may be in generating soluble DNA condensates that can be formulated within a gene-activated matrix intended for implantation in which targeting is not necessary and spontaneous transfection of infiltrating cells is desired. ${ }^{25}$ Alternatively, the greater stability of PEG-VS-CWK ${ }_{18}$ may result in its utility in modifying the surface of DNA condensates used during intravenous gene delivery.

Future studies will demonstrate the utility of PEGpeptides in gene activated matrixes and in altering the biodistribution of DNA condensates in vivo. The ability to form DNA cocondensates that incorporate both PEG and targeting ligands attached to $\mathrm{CWK}_{18}$ may provide a unique approach to systematically optimize gene delivery formulations for maximum efficacy in vivo.

\section{References and Notes}

1. Zhang, Y. P.; Reimer, D. L.; Zhang, G.; Lee, P. H.; Bally, M. B. Self-Assembling DNA-Lipid Particles for Gene Transfer. Pharm. Res. 1997, 14, 190-196.

2. Wu, G. Y.; Wu, C. H. Evidence for Targeted Gene Delivery to HepG2 Hepatoma Cells in Vitro. Biochemistry 1988, 27, 887-892.

3. Ogris, M.; Steinlein, P.; Kursa, M.; Mechtler, K.; Kircheis, R.; Wagner, E. The Size of DNA/Transferrin-PEI complexes is an Important Factor for Gene Expression in Cultured Cells. Gene Ther. 1998, 5, 1425-1433.

4. Tang, M. X.; Redemann, C. T.; Szoka, F. C., J r. In Vitro Gene Delivery by Degraded Polyamidoamine Dendrimers. Bioconjugate Chem. 1996, 7, 703-714.

5. Niven, R.; Pearlman, T.; Wedeking, T.; Mackeigan, J .; Noker P.; Simpson-Herren, L.; Smith, J. G. Biodistribution of Rádiolabeled Lipid-DNA' Complexes and DNA in Mice. J. Pharm. Sci. 1996, 87, 1292-1299.

6. Nishikawa, M.; Takemura, S.; Takakura, Y.; Hashida, M. Targeted Delivery of Plasmid DNA to Hepatocytes In Vivo: Optimization of the Pharmacokinetics of Plasmid DNA/ Galactosylated Poly (L-Lysine) Complexes by Controlling their Physicochemical Properties. J . Pharm. Exp. Ther. 1998, 287, 408-415.

7. Kwoh, D. Y.; Coffin, C. C.; Lollo, C. P.; J ovenal, J .; Bananszczyk, M. G.; Mullen, P.; Phillips, A.; Amini, A.; Fabriycki, J .; Bartholomew, R. M.; Brostoff, S. W.; Carlo, D. J. Stabilization of Poly-L-Lysine/DN A Polyplexes for In Vivo Gene Delivery to the Liver. Biochim. Biophys. Acta 1999, 1444, 171-190.

8. Woodle, M. C. Controlling Liposome Blood Clearance by Surface-Grafted Polymers. Adv. Drug Delivery Rev. 1998, 32, 139-152.

9. Torchilin, V.; Omelyanenko, V. G.; Papisov, M. I.; Bogdanov, A. A.; Trubetskoy, V. S.; Herron, J . N.; Gentry, C. A. Poly(ethylene glycol) on the Liposome Surface: On the Mechanism of Polymer-Coated Liposome Longevity. Biochim. Biophys. Acta 1994, 1195, 11-20. 
10. Torchilin, V. P. Polymer-Coated Long-Circulating Microparticulate Pharmaceuticals. J. Microencaps. 1998, 15 (1), 1-19.

11. Wolfert, M. A.; Schacht, E. H. Toncheva, V: Ulbrick, K. Nazarova, O.; Seymour, L. W. Characterization of Vectors for Gene Therapy Formed by Self-Assembly of DNA with Synthetic Block Co-Polymers. Human Gene Ther. 1996, 7, 2123-2133.

12. Toncheva, V.; Wolfert, M. A.; Dash, P. R.; Oupicky, D.; Ulbrich, K.; Seymour, L. W.; Śchacht, E. H. Novel Vectors for Gene Delivery Formed by Self-Assembly of DNA with Poly(L-lysine) Grafted with Hydrophobic Polymers. Biochim. Biophys. Acta 1998, 1380, 354-368.

13. Choi, Y. H.; Liu, F.; Kim, J. K.; Choi, Y. K.; Park, J . S.; Kim, S. W. Polyethylene Glycol-Grafted Poly-L-Lysine as Polymeric Gene Carrier. J . Controlled Rel ease 1998, 54, 39-48.

14. Choi, J. S.; Lee, E. J .; Choi, Y. H.; J eong, J. Y.; Park, J . S Poly(ethylene glycol)-block-poly(L-lysine) Dendrimer: Novel Linear Polymer/Dendrimer Block Copolymer Forming a Spherical Water-Soluble Polyionic Complex with DNA. Bioconjugate Chem. 1999, 10, 62-65.

15. Harada, A.; Kataoka, K. Formation of Polyion Complex Micelles in an Aqueous Milieu from a Pair of OppositelyCharged Block Copolymers with Poly(ethylene glycol) Segments. Macromol ecules 1995, 28, 5294-5299.

16. Katayose, S.; Kataoka, K. Water-Soluble Polyion Complex Associates of DNA and Poly(ethylene glycol)-Poly(L-lysine) Block Copolymers. Bioconjugate Chem. 1997, 8, 702-707.

17. Katayose, S.; Kataoka, K. Remarkable Increase in Nuclease Resistance of Plasmid Supramolecular Assembly with Poly(ethylene glycol)-Poly(L-lysine) Block Copolymer. J . Pharm. Sci. 1998, 87, 160-163.

18. Plank, C.: Zatloukal, K.; Cotten, M.; Mechtler, K.; Wagner, E. GeneTransfer into Hepatocytes Úsing Asialoglycoprotein Receptor Mediated Endocytosis of DNA Complexed with an Artificial Tetraantennary Galactose Ligand. Bioconjugate Chem. 1992, 3, 533-539.
19. Wadhwa, M. S.; Collard, W. T.; Adami, R. C.; McKenzie, D. L.; Rice, K. G. Peptide-M ediated Gene Delivery: Influence of Peptide Structure on Gene Expression. BioconjugateChem. 1997, 8, 81-88.

20. Burns, J. A.; Butler, J. C.; Moran, J.; Whitesides, G. M. Selective Reduction of Disulfides by Tris-(2-carboxyethyl)phosphine. J . Org. Chem. 1991, 56, 2648-2650.

21. Bradford, M. M. A Rapid and Sensitive Method for the Quantitation of Microgram Quantities of Protein Utilizing the Principle of Protein-Dye Binding. Anal. Biochem. 1976, $72,248-254$.

22. Teribesi, J .; K wok, K. Y.; Rice, K. G. I odinated Plasmid DNA as a Tool for Studying Gene Delivery. Anal. Biochem. 1998, 263, 120-123.

23. Pouton, C. W.; Seymour, L. W. Key Issues in Non-Viral Gene Delivery. Adv. Drug Delivery Rev. 1998, 34, 3-19.

24. Wadhwa, M. S.; Knoell, D.; Y oung, T.; Rice, K. G. Targeted Gene Delivery with a Low Molecular Weight Glycopeptide. Bioconjugate Chem. 1995, 6, 283-291.

25. Fang, J.; Zhu, Y. Y.; Smiley, E.; Bonadio, J .; Rouleau, J .; Goldstein, S. A.; McCauley, L. K.; Davidson, B. L.; Roessler, B. J . Stimulation of New Bone F ormation by Direct Transfer of Osteogenic Plasmid Genes. Proc. Natl. Acad. Sci. 1996, $93,5753-5758$

\section{Acknowledgments}

The authors acknowledge financial support provided by $\mathrm{NIH}$ grants GM 48049, DE 13004 and support from Selective Genetics, Inc.

J S990072S 